\section{Optical clock recovery scheme for a high bit rate nonreturn-to-zero signal using fiber Bragg grating filters}

\author{
Jaemyoung Lee \\ Korea Polytechnic University \\ 2121 Jungwang-dong Shihung-si \\ Kyunggi-do, Korea 429-793 \\ E-mail: lee@kpu.ac.kr
}

\author{
Hyunwoo Cho \\ Sang-Kyu Lim \\ Sang Soo Lee \\ Je Soo Ko \\ Electronics and Telecommunications \\ Research Institute \\ 161 Gajeong-dong Yuseong-gu \\ Daejeon, Korea 305-350
}

\begin{abstract}
For optical clock recovery of nonreturn-to-zero (NRZ) signals more than $40 \mathrm{~Gb} / \mathrm{s}$, we propose and experimentally demonstrate a simple clock recovery scheme using beat processing. Through the proposed scheme, we square the adjustment range of the variable optical attenuator (VOA) and achieved an enhanced clock-to-noise ratio (CNR) of more than $15 \mathrm{~dB}$ in the experiment, compared to a system without the proposed scheme. () 2005 Society of Photo-Optical Instrumentation Engineers. [DOI: 10.1117/1.1849592]
\end{abstract}

Subject terms: optical clock recovery; nonreturn to zero; fiber Bragg grating filter; variable optical attenuator.

Paper L040541R received Aug. 13, 2004; revised manuscript received Sep. 29, 2004; accepted for publication Oct. 18, 2004; appeared online Nov. 2, 2004; published online Feb. 4, 2005.

\section{Introduction}

High-speed optical time division multiplexed communications require efficient optical clock recovery technologies at receiver units. ${ }^{1}$ Many techniques have been proposed and developed for optical clock recovery, but most of them have focused on a return-to-zero (RZ) signal. ${ }^{2,3}$ Since a nonreturn-to-zero (NRZ) signal theoretically does not have the clock component, most schemes have employed a preliminary signal process to recover the clock of the signal. ${ }^{4,5}$ However, modulated optical NRZ signals show the clock components, due to the limited bandwidths of devices used in the system. 6,7

Using one of the clock components and the carrier frequency component, we propose a cost-effective and simple clock recovery scheme through beat processing. The proposed scheme recovers the clock frequency by extracting two frequency components of the optical spectrum that are harmonically related to the clock frequency, equalizing them for the best clock-to-noise ratio (CNR), and then beating them to recover the clock frequency. In addition, we experimentally demonstrate the optical clock recovery of a 40-Gb/s NRZ signal. To extract two frequency components for the beat processing, instead of a tunable filter as in Ref. 6 , we used two fiber Bragg grating (FBG) filters, of which each reflection frequency corresponds to the carrier frequency and to one of the clock frequency components. The reason for using two wavelength-fixed FBG filters is that the carrier frequency and clock frequencies of a signal are determined by the wavelength of the light source, bit rate, and modulation format, which do not change for a given transmission system. For a maximum CNR, the amplitude of the carrier frequency component was adjusted to that of the extracted clock frequency component by a variable optical attenuator (VOA) by referring to an optical spectrum analyzer.

\section{Operation of the Proposed Scheme}

Figure 1 shows the proposed scheme for optical clock recovery, which consists of an optical circulator, a VOA, and two FBG filters. A signal is injected into the first FBG filter (OF1) via the optical circulator. One of the clock frequency components is reflected back to the circulator by the OF1. The rest of the frequency components propagating toward the second FBG filter (OF2) lose their optical power through the VOA. The amplitude of the carrier frequency component sent back to the circulator by the OF2 is reduced again while it passes to the circulator through the VOA. By adjusting the VOA, the amplitude of the carrier frequency component reflected back by the OF2 can be made equal to that of the clock frequency component extracted by the OF1. In the proposed scheme, the VOA can extend its adjustment range up to the second power of its maximum attenuation value, because the carrier frequency component experiences optical losses twice by the VOA as it propagates toward the $\mathrm{OF} 2$ and back to the circulator after being reflected by the OF2 through the VOA.

\section{Experiment and Results}

We examined the validity of the proposed scheme shown in Fig. 1 for the 40-GHz optical clock recovery from a 40$\mathrm{Gb} / \mathrm{s}$ NRZ signal. From the 40-Gb/s signal, which was produced by multiplexing four $10-\mathrm{Gb} / \mathrm{s}$ pseudorandom binary sequences with data of $2^{31}-1$, two frequency components were extracted with equal amplitude to generate a beat signal with a frequency equal to the bit rate of the $40-\mathrm{Gb} / \mathrm{s}$

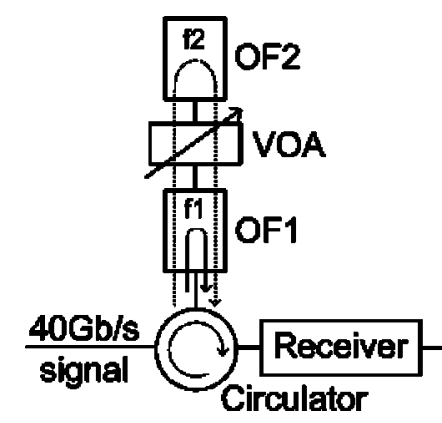

Fig. 1 Proposed optical clock recovery scheme for NRZ and experiment setup for $40-\mathrm{GHz}$ clock recovery. 

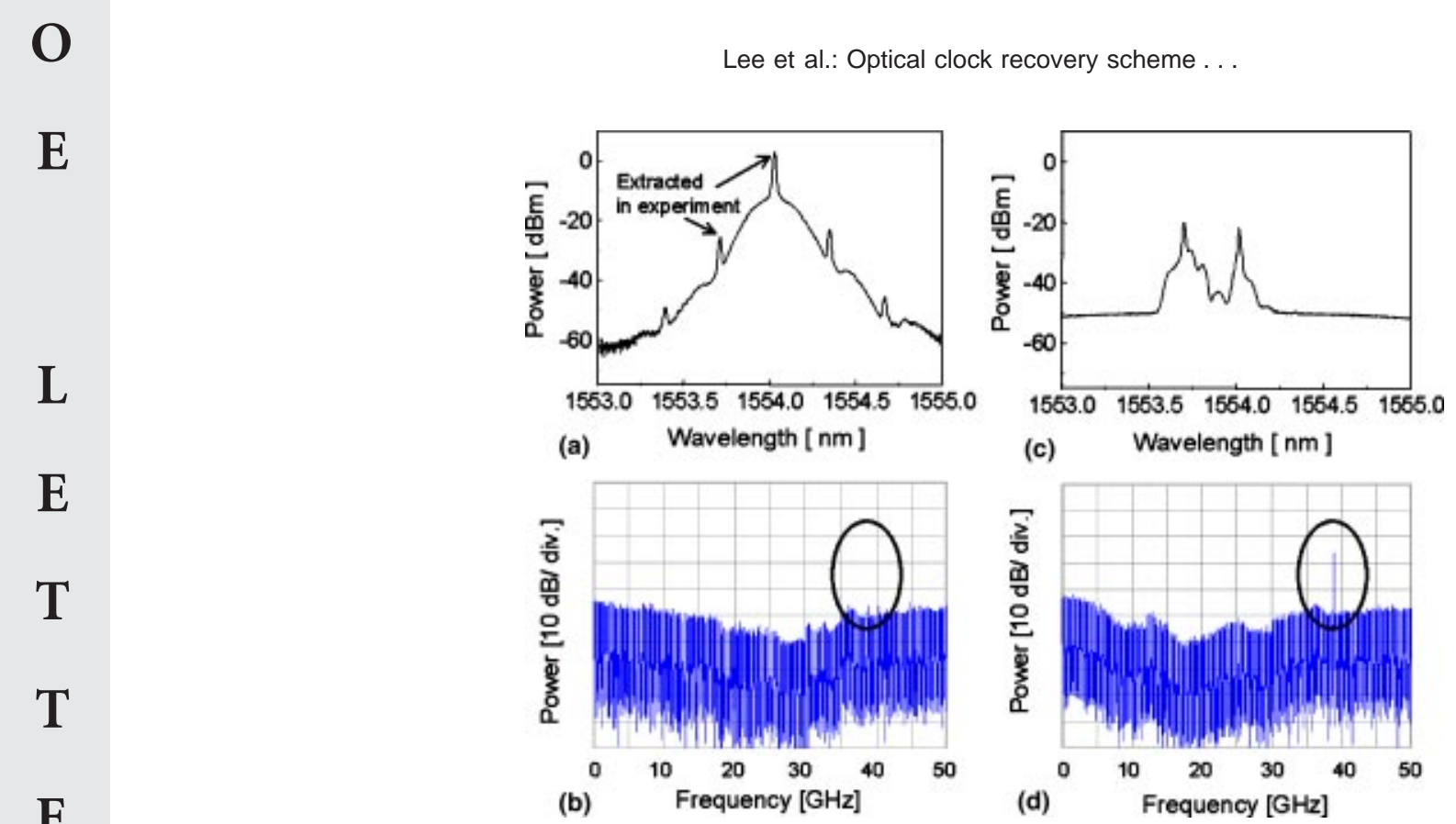

Fig. 2 Optical and rf spectra of 40-Gb/s signal. (a) Optical spectrum and (b) if spectrum of the input $40-\mathrm{Gb} / \mathrm{s}$ NRZ signal. (c) Optical spectrum and (d) rf spectrum at the output of the circulator.

NRZ signal through two FBG filters and a VOA. The optical and rf spectra of the applied $40-\mathrm{Gb} / \mathrm{s}$ signal is shown in Figs. 2(a) and 2(b), respectively. Note that two sidebands at intervals equal to the bit rate from the carrier frequency are observed in the optical spectrum. One of these clock components and the carrier frequency component were extracted with equal amplitude from the 40-Gb/s signal through the two FBG filters and a VOA. Other frequency components were suppressed, since they are out of the reflection regions of the filters. Two extracted frequency components with equal amplitude are mixed at a receiver in which a beat signal with a frequency of $40 \mathrm{GHz}$ is generated. The frequency of the generated beat signal is the frequency difference between the two extracted frequency components, which is the clock frequency in the transmitter of the system. Figures 2(c) and 2(d) show the optical and rf spectra of the generated beat signal at the output of the circulator. These figures show two equal amplitude peaks spaced $40 \mathrm{GHz}$ apart in the optical spectrum, and the enhanced clock frequency component in the rf spectrum.

Through beat processing in the proposed clock recovery scheme, the rf power of the clock frequency component [Fig. 2(d)] increased more than $15 \mathrm{~dB}$ at the same optical mean power of $0 \mathrm{dBm}$ before the receiver, compared with the rf spectrum without beat processing [Fig. 2(b)], and was about $15 \mathrm{~dB}$ larger than the residual spectra [Fig. 2(d)].

\section{Conclusion}

We propose a simple and cost-effective scheme for clock recovery for high-speed optical transmission systems through beat processing using FBG filters and a VOA. In addition, we experimentally demonstrate the optical clock recovery of $40-\mathrm{Gb} / \mathrm{s}$ NRZ data. To generate a beat signal, two frequency components spaced $40 \mathrm{GHz}$ apart in the optical spectra are extracted and equalized in amplitude by a VOA, the adjustment range of which is squared in the proposed scheme. Through the beat processing, the proposed scheme enhances the clock frequency component of the modulated NRZ signal. We expect that the proposed scheme can operate as an efficient clock extraction system for high-speed optical communications.

\section{References}

1. N. Hanik, A. Ehrhardt, A. Gladisch, C. Peucheret, P. Jeppesen, L. Molle, R. Freund, and C. Caspar, "Extension of all-optical networktransparent domains based on normalized transmission sections," $J$. Lightwave Technol. 22(6), 1439-1453 (2004).

2. B. Sartorius, C. Bornholdt, O. Brox, H. J. Ehrke, D. Hoffmann, R. Ludwig, and M. Mohrle, "All-optical clock recovery module based on self-pulsating DFB laser," Electron. Lett. 34(17), 1664-1665 (1998).

3. D. T. K. Tong, B. Mikkelsen, T. N. Nielsen, K. F. Dreyer, and J. E. Johnson, "Optoelectronic phase-locked loop with balanced photodetection for clock recovery in high-speed optical time-divisionmultiplexed systems," IEEE Photonics Technol. Lett. 12(8), 10641066 (2000).

4. W. Mao, Y. Li, M. Al-Mumin, and G. Li, "All-optical clock recovery for both RZ and NRZ data," IEEE Photonics Technol. Lett. 14(6), 873-875 (2002)

5. Y. D. Jeong, H. J. Lee, H. Yoo, and Y. H. Won, "All-optical NRZ-toPRZ converter at $10 \mathrm{~Gb} / \mathrm{s}$ based on self-phase modulation of FabryPerot laser diode," IEEE Photonics Technol. Lett. 16(4), 1179-1181 (2004).

6. B. Franz, "Optical signal processing for very high speed (>40 Gbit/s) ETDM binary NRZ clock recovery," Optical Fiber Communication 2001, Anaheim, CA, Paper MG1 (2001).

7. T. Tokle, C. Peucheret, and P. Jeppesen, "Advanced modulation formats in $40 \mathrm{Gbit} / \mathrm{s}$ optical communication systems with $80 \mathrm{~km}$ fibre spans," Opt. Commun. 225, 79-87 (2003). 